\title{
The HPS experiment at JLab
}

\author{
Marzio De Napoli ${ }^{1, a}$ for the HPS Collaboration \\ ${ }^{1}$ INFN - Sezione di Catania, Via Santa Sofia 64, 95123 Catania, Italy
}

\begin{abstract}
Many Beyond-Standard-Model theories predict a new massive gauge boson, such as a "dark" or "heavy photon". The heavy photon is expected to mix with the Standard Model photon through kinetic mixing and therefore have a small coupling to electric charge. The Heavy Photon Search (HPS) experiment is searching for a heavy photon at the Thomas Jefferson National Accelerator Facility (JLab), in the mass range $20-500 \mathrm{MeV} / \mathrm{c}^{2}$. In particular HPS looks for the $e^{+} e^{-}$decay channel of heavy photons radiated by electron Bremsstrahlung, employing both an invariant mass search and detached vertexing techniques. The experiment employs a compact forward spectrometer comprising silicon microstrip detectors for vertexing and tracking and an electromagnetic calorimeter for particle identification and triggering. HPS took data successfully in 2015 and 2016 at $1.05 \mathrm{GeV}$ and $2.3 \mathrm{GeV}$ beam energies, respectively. First results are expected to be presented soon.
\end{abstract}

\section{Introduction}

The existence of an additional U(1) gauge symmetry in nature is predicted by several beyond the Standard Model theories [1,2]. The associated gauge boson $A^{\prime}$, aka "dark" or "heavy photon", can kinematically mix with the photon through quantum loops of heavy particles that carry both the standard model hypercharge and the dark force equivalent [3, 4]. This kinetic mixing results in an effective interaction $\epsilon e A_{\mu}^{\prime} J_{E M}^{\mu}$ of the $A^{\prime}$ to the electromagnetic current $J_{E M}^{\mu}$, suppressed relative to the electron charge $e$ by a factor $\epsilon \leq 10^{-3}$. Since heavy photons couple to electrons, they can be produced by electron Bremsstrahlung off heavy nuclear targets and can decay to $e^{+} e^{-}$pairs.

HPS is an experiment to search for $A^{\prime}$ in fixed target electroproduction at Hall B of the JLab. The high duty-cycle, high-quality and high-intensity CEBAF electron beam is crucial to generate large integrated luminosities while keeping beam-related background under control. The electron beam, of intensity between $50 \mathrm{nA}$ and $400 \mathrm{nA}$, impinging on $0.15 \%-0.25 \%$ radiation length tungsten foils, can produce heavy photons through a process analogous to Bremsstrahlung, and the $A^{\prime}$ can then decay to $e^{+} e^{-}$ pairs (Fig. 1). Since the coupling of heavy photons to $e^{+} e^{-}$ pairs is much weaker than the electromagnetic coupling, heavy photon production is buried under a background of pairs from virtual photons. By reconstructing the momenta of the $e^{+} e^{-}$pairs, HPS is searching for a narrow resonance in the invariant mass spectrum over the quantum electrodynamics (QED) background (the traditional "bump-hunting"). The main QED background reactions are the radiative trident production and the Bethe-Heitler process (Fig. 1). Although the Bethe-Heitler process has

\footnotetext{
a e-mail: marzio.denapoli@et.infn.it
}

much larger total cross-section than the radiative one, it can be significantly reduced with appropriate kinematic cuts. Instead, the radiative trident process is an irreducible background, with the same kinematics as the signal in a small invariant-mass window centered on the A' mass. The signal is characterized by a very-forward emitted $A^{\prime}$ that carries most of the beam energy $E$. Therefore, the $A^{\prime}$ decay products are boosted forward with typical polar angles $\sim M_{A^{\prime}} / E$, of the order of few degrees. Moreover, depending on $M_{A^{\prime}}$ and $\epsilon, A^{\prime}$ can be long-lived, with a distinct secondary decay vertex detached from the target. HPS is also using this signature to distinguish the signal from the $\mathrm{QED} e^{+} e^{-}$pairs promptly produced in the target.

\section{The HPS Experiment}

The HPS detector was designed to match the experimental requirements dictated by the search strategies and the signal kinematics discussed above. Resonance search requires excellent track reconstruction for electrons and positrons with a good angular and momentum resolution, which is most easily accomplished with charged particle spectrometry. Detached vertexing requires the tracking detectors to be placed just downstream of the target to minimize extrapolation errors and optimize vertex resolution. Detectors must be also placed as close to the beam direction as possible to cover with high acceptance the forward angular region.

The proximity of the tracking detectors to the beam dictates stringent requirements on acceptable beam size, stability, and halo. At the same time it necessitates protection collimation and real time monitoring and circuitry to protect against errant beam motion. During the data taking in 2015 and 2016 high quality beam was delivered and 
a)

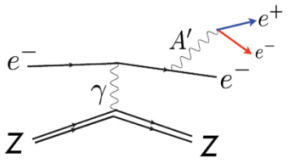

b)

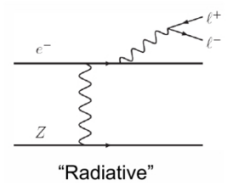

c)

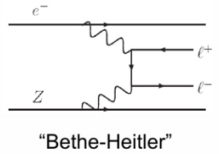

Figure 1. (a) Feynman diagram for radiative $A^{\prime}$ electroproduction and $A^{\prime}$ decay to $e^{+} e^{-}$pairs. Diagrams of radiative $(b)$ and Bethe-Heitler $(c)$ QED reactions, the primary background for the $A^{\prime}$ search, are also shown.

the beam feedback and fast shut down systems described in [5] worked in maintaining the beam position stable and protecting the silicon sensors from errant beam exposure. The HPS apparatus is split into symmetric upper and lower halves since the detectors must avoid a region of intense electron flux caused by the small angle multiple coulomb scattering in the target and the horizontal dispersion in the magnetic field of the analyzing magnet of the electrons which have radiated energy in the target. The resulting "dead zone" has an angular range of $\theta_{y}<15 \operatorname{mrad}(y$ is the vertical dimension). In addition, the beam is transported in vacuum through the tracker to minimize beam-gas interaction backgrounds. The detector setup is shown in Fig. 2. It is made of $\mathrm{a} \sim 1 \mathrm{~m}$ long Silicon Vertex Tracker (SVT) inside an analyzing dipole magnet to reconstruct charged particle trajectories and vertexes, and a fast lead-tungstate electromagnetic calorimeter (ECal) to measure particle energies, identify the $e^{+} e^{-}$pairs, and generate the trigger of the experiment.

The SVT is made of 6 layers of pairs of closely-spaced silicon microstrip sensors mounted back-to-back to form a module. A small stereo angle between the two layers (50 or $100 \mathrm{mrad}$ ) provides three-dimensional tracking and vertexing. The SVT material budget in the tracking volume is minimized to reduce multiple scattering and opti- mize both the invariant mass and vertex resolutions that directly affect the experimental sensitivity. The sensors have an active area of $98.33 \times 38.34 \mathrm{~mm}^{2}$, they are 320 $\pm 20 \mu \mathrm{m}$ thick and have a sense (readout) pitch of 30 (60) $\mu \mathrm{m}$. To achieve large acceptance and good vertexing resolution, the first layer of silicon detectors is placed just 10 $\mathrm{cm}$ downstream of the target with the sensor edges only $500 \mu \mathrm{m}$ above and below the beam. Sensors are readout by the APV25 chip [6] with a custom DAQ system based on the Advanced Telecom Communications Architecture (ATCA) technology.

The ECal [7] is made of $442 \mathrm{PbWO}_{4}$ crystals, $16 \mathrm{~cm}$ thick, with a $13.3 \times 13.3 \mathrm{~mm}^{2}\left(16 \times 16 \mathrm{~mm}^{2}\right)$ front (rear) face. In each half of the detector, crystals are arranged in five rows of 46 crystals, except the one closest to the beam that has only 37 crystals. The light from each crystal, approximately 120 photons/MeV, is read out by largearea Avalanche PhotoDiodes (APD) glued to the back face. Signals from the APDs are read and amplified using custom-made pre-amplifiers before being sent to the JLab Flash ADC boards for digitization and processing. The onboard FPGA processes the digitized samples to provide information about the energy and the timing associated with each hit. This information, available during data-taking, is exploited by an advanced triggering algorithm matched to the foreseen $A^{\prime}$ signal.

The data collected in 2015 and 2016 demonstrated excellent performances of both the detectors. The SVT and Ecal primary goals of providing an high quality vertexing and tracking and a fast trigger in a large background environment were fully achieved. We anticipate first results from the brief $20151.1 \mathrm{GeV}$ run in early 2017.

\section{References}

[1] Feldman D., Kors B. and Nath P., Phys. Rev. D 75, 023503 (2007)

[2] Andreas S., Goodsell M. and Ringwald A., Phys. Rev. D 87, 025007 (2013)

[3] Galison P. and Manohar A., Phys. Lett. B 136, 279 (1984)

[4] Holdom B., Phys. Lett. B 166, 196 (1984)

[5] N. Baltzell et al., arXiv:1612.07821

[6] French M., Jones L., Morrissey Q., Neviani A., Turchetta R. et al., Nucl. Instr. and Meth. A 466, 359-65 (2001)

[7] Balossino I. et al., Nucl. Instr. and Meth. A 854, 89-99 (2017) 


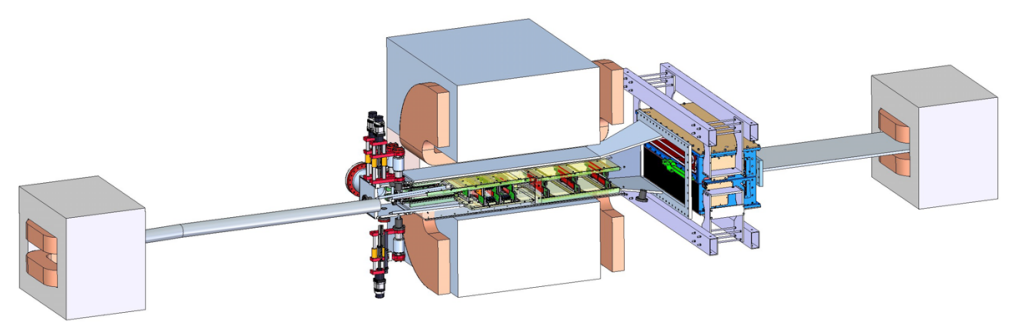

Figure 2. Layout of the HPS detector setup. From left to right: the first dipole magnet deflects the beam toward the target placed at the entrance of the second analyzing dipole magnet, the SVT inside the analyzing magnet, the ECal and the third dipole magnet to steer back the beam toward the beam dump. 